$J J M L L$

\title{
Le Narratif dans le Texte Dramatique: Une Médiation Narratoriale
}

\author{
Bashar Sami Yashooa \\ Département de français, Université Mustansiriyah, Irak
}

Received on: 8-8-2019

Accepted on: 16-12-2019

\section{Résumé}

Les premières notions des anciens, qui traitent l'analyse du récit et du texte théâtral, partent d'une distinction entre Mimésis, art de montrer, et Diégésis, art de raconter. Le théâtre se voit en général par l'aspect mimétique de sa construction. L'histoire est représentée sans être racontée ou narrée. Les recherches littéraires, ainsi que les critiques des anciens, tels d'Aristote et de Platon, paraissent limiter le théâtre au mode mimétique.

Cette limitation ne parait complètement pas effective au monde théâtral. Le script (texte dramatique) n'est qu'une traduction narrative d'une performance (représentation théâtrale). Un rapport entre les notions du texte théâtral et le récit pourrait ainsi s'établir. Notre problématique réside dans le fait que le texte dramatique, ou bien théâtral, pourrait inclure des aspects de narrativité à partir de principe fondamental des fonctions du narrateur et de l'instance narrative. La recherche d'un narrateur au texte théâtral est ainsi une nécessité.

Des instances narratoriales, autres que celles dramatiques des personnages, encadrent les actions et dirigent le lecteur vers une réception complète de l'œuvre. Le discours des personnages est une instance dans un niveau narratif autre que celle du narrateur. Ce narrateur qui occupe le plus souvent une position extradiégétique, crée un cadre narratif qui tâche d'accomplir le monde dramatique.

Les didascalies et les écrits paratextuels sont des registres énonciatifs constituant une instance autonome. Les actions, les sentiments et les expressions de visage sont effectivement muets, mais ils trouvent dans cette instance narratoriale du narrateur un outil de mettre en scène toute l'ambiance dramatique.

Le texte dramatique, ou bien théâtral, pourrait ainsi inclure des aspects de narrativités à partir d'un narrateur qui remplit des fonctions de médiation narrative. Il établit effectivement des affinités avec la narration et la description afin de rapporter le cadre et l'ambiance non verbale.

Mots-clés : Narrateur, théâtre, Diégésis, incipit, didascalie, paratexte.

\section{The Narrative in the Dramatic Text: A Narratorial Mediation}

\begin{abstract}
The first notions of the ancients, who deal with the analysis of the narrative and the theatrical text, start from a distinction between Mimesis, art of showing, and Diégesis, art of telling. The theater is generally seen by the mimetic aspect of its construction. The story is portrayed without being told. Literary research, as well as critics of the ancients, such as Aristotle and Plato, seem to limit the theater to mimetic mode.
\end{abstract}

${ }^{\odot} 2020$ JJMLL Publishers/Yarmouk University. All Rights Reserved. 
This limitation does not seem completely effective in the theatrical world. The script (dramatic text) is only a narrative translation of a performance (theatrical performance). A connection between the notions of the theatrical text and the story could thus be established. Our problem lies in the fact that the dramatic or theatrical text could include aspects of narrativity based on the fundamental principle of the functions of the narrator and the narrative instance. The search for a narrator with a theatrical text is a necessity.

Narrative instances, other than the dramatic ones of the characters, frame the actions and direct the reader towards a complete reception of the work. The characters' speech is an instance in a narrative level other than that of the narrator. This narrator, who most often occupies an extradiegetic position, creates a narrative framework that strives to accomplish the dramatic world.

Didascalies and paratextual writings are enunciative registers constituting an autonomous body. Actions, feelings and facial expressions are indeed silent, but they find in this narratorial instance of the narrator a tool to stage the whole dramatic atmosphere.

The dramatic or theatrical text could thus include aspects of narrativities from a narrator who fulfills functions of narrative mediation. It effectively establishes affinities with the narration and the description in order to relate the framework and the non-verbal atmosphere.

Keywords: Narrator, theater, Diégesis, incipit, didascalie, paratext.

\section{Introduction}

La narration est principalement l'action de raconter une histoire, une intrigue ou bien un simple événement. Le concept de narrer dans un cadre théâtral est apparemment éloigné des études de la narratologie. L'analyse du texte théâtral et du simple récit part des premières notions de l'analyse littéraire des anciens, Platon et Aristote, qui opposent, à des degrés différents, Diégesis, art de raconter, à Mimésis, art de monter.

Le théâtre est ainsi marqué par l'aspect mimétique de sa construction de base. L'histoire est représentée en direct sans apparemment être racontée ou narrée. Les recherches littéraires, ainsi que les critiques modernes et contemporaines des années soixante, paraissent limiter la narration au monde diégétique.

L'absence de médiateur narratif dans le genre dramatique est un point sur lequel insistent certains critiques du XXème et du XXIème siècle. Genette, un theoricien d'analyse narrative de la littérature française moderne, parait, dans ses Figures, plutôt s'engager aux cotés de la narration limitée au récit. Richardson qui chereche un narrateur dans le théâtre, Ricœur qui délimite la narration au recit, Ubersfeld qui parle d'un discours de l'auteur dans le théâtre, entre autres, nous ouvrent un volet d'une analyse narrative dans le texte dramatique.

Il est question de chercher le fait que le texte dramatique, ou bien théâtral, pourrait inclure des aspects de narrativité à partir de principe fondamental des fonctions du narrateur et de l'instance narrative. La recherche d'un narrateur dans le texte théâtral est le point de départ de notre analyse structurale et dramatique. 
Dans la deuxième moitie du XXieme siècle, la narratologie se révèle comme discipline du récit. Cet article tente de retracer certaines pistes narratives dans les écrits dramatiques considérées comme exclus des études narratives. Quel rapport pourrait-il s'établir entre les notions du récit et le texte théâtral ? Le théâtre, art de représentation direct, pourrait-il, en tant que mimésis, détenir des éléments fondamentaux du récit, voire diégésis?

Les analyses de tout texte dramantque révèlent effectivement des personnages chargés des répliques à la représentation textuelle des actions théâtrales. Mais qui est-ce qui nous les présente ? Comment faire s'il ne s'agit pas, dans le texte dramatique, de répliques ? Mais d'une action, d'une expression de visage, d'un geste ou d'un mouvement ! Quelle possibilité reste-il ainsi dans le texte théâtral ?

\section{Le théâtre entre Diégésis et Mimésis}

Genette, avant d'entreprendre sa distinction entre mimésis et diégésis, a exposé les propos d'Aristote, de Platon et de Socrate, les grands théoriciens de la littérature.

Aristote dans sa poétique, ainsi que Platon dans son troisième livre de la république, ont défini les deux modes d'imitation poétique (mimésis, diégésis) :

Pour Platon, le domaine de ce qu'il appelle lexis (ou façon de dire, par opposition à logos, qui désigne ce qui est dit) se divise théoriquement en imitation proprement dite (mimésis) et simple récit (diégésis). Par simple récit, Platon entend tout ce que le poète raconte « en parlant en son propre nom, sans essayer de nous faire croire que c'est un autre qui parle. (Genette 1969, 50)

Ainsi, à la diégésis, le narrateur parle en son nom et ne cache pas sa présence mediatrice. Le lecteur sait bien que l'histoire est racontée et médiée par un ou plusieurs narrateurs. La narration n'est pas masquée, l'histoire est narrée, médiée par du langage. Par contre à la mimésis, l'histoire parait se raconter elle - même, sans médiation, sans narrateur apparent. C'est (montrer) qui se trouve clairement dans le thêâtre, dans le drame, dans certains romans dialogués ou monologués pour construire ainsi l'impression d'une présence immédiate de la fiction (Genette 1972, 184-191). C'est comme une distinction entre la poésie narrative et la poésie dramatique. Socrate voit que toute imitation est un récit. Mais il y a, pour lui, des aspects de représentation directe, comme le dialogue qui se trouve dans des textes dramatiques. L'histoire a donc traité le principe de l'imitation. Aristote et Platon trouvent que diégésis et mimésis sont deux modes d'imitation poétique, alors que Socrate trouve que le récit s'oppose à l'imitation et se déclare comme son antithès (Genette 1969, 50).

Le principe d'Aristote n'est pas loin de celui de Platon. Pour lui, toute poésie est ramenée à l'imitation qui se distingue des deux modes narratifs ; le direct, nommé par Platon imitation ou mimésis, et l'indirect que nomme Platon diégésis. Aristote identifie le genre dramatique au mode imitatif malgré son caractère mixte. Le genre épique, contenant des dialogues et discours au style direct ainsi qu'une partie narrativisée, voire récit, demeure narratif bien que la partie imitative dépassela partie narrative qui encadre l'imitatif (dialogue, discours direct), et sert du fond et de la trame au discours. Homère,par 
exemple, intervient le moins possible en personne dans son poème. Le caractère mixte, imitatif et narratif du genre épique homérique explique l'aspect narratif à son fond, mais dramatique à sa grande étendue. Il n'y a donc pas de différence entre les deux classifications d'Aristote et de Platon. C'est une simple variante de terme qui exprime le même essentiel (Genette 1969, 52).

Genette a eu, de sa part, une vision peu différente de celle de Platon et d'Aristote. Tant que l'imitation directe,selon les deux théoriciens, se rapporte aux gestes et aux paroles, comment l'on peut les représenter en littérature!

Les actions échappent au plan linguistique, seule activité de poète. La parole des personnages n'est pas proprement représentative, puisque l'imitation directe des paroles est une reproduction littérale de tel ou tel discours ou parole des personnages. Homère, par exemple, ne représente pas dans l'Iliade le discours de Chryses, il le répète littéralement, et le constitue s'il s'agit d'un discours fictif (Genette 1969, 53). Ainsi, dans le cas de la diction de parole ou de geste, il n’y a pas de représentation.

Le récit met en œuvre des effets de distance, il crée un mode narratif qui règle l'information au lecteur (Genette 1972, 184). Selon Genette, tout récit est diégésis (raconter), et laisse l'impression de mimésis (imiter) à partir de l'idée que derrière tout récit il y a un narrateur : "Le récit ne "représente" pas une histoire (réelle ou fictive), il la raconte, c'est-à-dire qu'il la signifie par le moyen du langage [...]. Il n’y a pas de place pour l'imitation dans le récit. (Genette 1983, 29).

Genette conclue ;

Le seul mode que connaisse la littérature en tant que représentation est le récit, équivalent verbal d'événements non verbaux et aussi (...) d'événements verbaux, sauf à s'effacer dans ce dernier cas devant une citation directe où s'abolit toute fonction représentative, à peu près comme un orateur judiciaire peut interrompre son discours pour laisser le tribunal examiner lui-même une pièce à conviction. La représentation littéraire, la mimésis des anciens, ce n'est donc pas le récit plus les "discours" : c'est le récit, et seulement le récit. Platon opposait mimésis à diégèses comme une imitation parfaite à une imitation imparfaite ; (...) l'imitation parfaite n'est plus une imitation, c'est la chose même, et finalement la seule imitation, c'est l'imparfaite. Mimésis, c'est diégèses. (Genette 1969, 55-56).

Ainsi, il voit entre diégésis et mimésis des degrés de diégésis, selon la présence du narrateur dans son récit en créant des éléments narratifs lors de son acte de raconter. Pour lui, un récit ne peut véritablement imiter la réalité ; c'est toujours un acte fictif de langage prévenu d'une instance narrative.

Certaines tendances contemporaines rejoignent le principe de l'imitation directe dans le théâtre et semblent le considérer, ainsi que ses écritures, comme exclu des principes de la narratologie moderne : "Le drame est sans médiation narratoriale (...) est mimétique plutôt que diégétique, agi plutôt que narrée » (KEIR, cité par Richardson 1988, 193) (je traduis).

Genette voit donc d'après son analyse des deux modes (Mimésis - Diégésis) que tout ce qui est littéraire appartient au monde diégétique sans compter la dominance du mode dramatique ou narratif, car tout ce qui est écrit se range aux côtés d'imitation médiatisée. 
En fait, assister à un spectacle est autre que le lire. C'est la différence entre le théâtre écrit et celui auquel nous assistons en direct. L'écrit trouve sa place avec le roman, le conte, la nouvelle, alors que le spectacle va plutôt vers le cinéma où l'on entend les répliques, voit l'action, le geste, et les caractères du corps sans médiation.

Par contre, le texte nous rapporte le discours qui exige une interprétation et un moyen narratif rapportant ce qui est muet et mimétique. C'est une incarnation des éléments non discursifs dans le monde textuel. Une voix encadre donc ce discours des personnages et nous transmet leurs actions et caractères.

Dans le théâtre, les spectateurs n'ont pas besoin de moyen pour identifier qui parle et qui fait son discours, car leurs yeux les dirigent vers l'énonciateur. Mais, dans texte dramatique, le besoin d'un guide qui nous désigne cet énonciateur est une nécessité. De maints exemples se trouvent dans des textes théâtraux dont voici un du médecin malgré lui de Molière :

SGANARELLE. - Et vous êtes un impertinent, de vous ingérer des affaires d'autrui : apprenez que Cicéron dit, qu'entre l'arbre et le doigt, il ne faut point mettre l'écorce12. (Ensuite il revient vers sa femme, et lui dit, en lui pressant la main) Ô çà faisons la paix nous deux. Touche là (Molière 2000, 24)

Il y a dans cette réplique deux énonciateurs ; Saganarelle en tant que personnage qui joue son rôle et dit son discours, et un autre qui insère son discours au milieu de la réplique afin de désigner la personne à laquelle Sganarelle s'adresse (sa femme) et lui dit ce qu'ils doivent faire. C'est la voix d'un narrateur qui encadre la réplique comme un récit-cadre encadrant le discours. Dans le théâtre, on n'a pas besoin de ce deuxième énonciateur tant qu'on voit à qui s'adresse le personnage. L'acte du langage du narrateur-cadre est organisateur du monde théâtral et ses composantes scéniques dans une narrativité mimétique (discours des personnages) et une narrativité diégétique (le récit des actes et des actions) :

Le discours direct n'est pas complètement autonome puisque le narrateur, on le voit, a besoin de reconstituer la situation d'énonciation pour que le message puisse être correctement interprété par le lecteur ; ce faisant, il interprète les intentions de celui qui parle (Buffard-Moret 2000, 40)

Nous adoptons ainsi le terme de (script) pour désigner le texte dramatique, et le terme de (performance) pour designer la représentation théâtrale. Le script est une traduction d'une performance théâtrale, les deux se retrouvent en deux niveaux différents ; logiquement, la représentation théâtrale est au niveau premier en tant que mimésis, alors que le script est au niveau second en tant que diégésis. Ils représentent deux instances différentes. Nous allons, à partir de ce principe d'imitation littéraire, essayer de prouver que le texte dramatique représente dans son fond, en dépit de son caractère mimétique, des éléments narratifs provenant d'une instance narratoriale.

\section{Les didascalies : une instance narrative}

Richardson parle d'un narrateur dans le théâtre, autre que les personnages qui peuplent la pièce. Il s'agit d'un narrateur qui encadre et raconte les actions des personnages (Richardson, 1988, 194). Dans le 
spectacle, le dramaturge est complètement coupé de ses personnages — acteurs et de son public. Le spectateur entend une série de discours et voit l'action, le lieu et comprend la situation spatio-temporelle où évolue l'intrigue et vivent les personnages sans aucun intermédiaire. Toute information sur le décor et l'attitude des personnages sont représentées devant lui. Mais, le texte dramatique comporte une couche discursive ; le discours narratif d'un narrateur afin d'accomplir le monde dramatique dans un registre énonciatif différent du registre dialogal.

Les couches textuelles, telles les didascalies et leurs éléments qui éclaircissent la situation de communication des personnages, déterminent les conditions d'énonciation des discours. Elles ont pour fonction d'orienter le sens des messages et de constituer des messages autonomes entre le dialogue et sa compréhension globale auprès du lecteur :

\section{ROBERT. Il passe ensuite vers le mari, qui, pareillement, lui parle toujours, en le faisant reculer, le frappe avec le même bâton, et le met en fuite, il dit à la fin.}

- Compère, je vous demande pardon de tout mon cœur, faites, rossez, battez, comme il faut, votre femme, je vous aiderai si vous le voulez (Molière 2000, 23)

Techniquement, les didascalies sont marquées textuellement par des traits qui les séparent du dialogue ; soit, elles sont mises en parenthèses, soit en italique, ce qui les visualise nettement du dialogue prononcé. Imaginons que cette réplique de M.Robert est produite sans l'indication du narrateur qui encadre le début de son discours, nous perdrons une partie importante des circonstances qui accomplissent la mise en scène de la séquence. L'acte de passer, de reculer, de frapper, de fuir et de dire sont représentés sur scènes, mais qui devront être transmis au simple lecteur textuellement par un moyen narratif dans un niveau différent de celui du dialogue.

Larthomas fait, dans son Langage dramatique, une distinction entre l'oral et l'écrit à travers le problème spécifique du théâtre. Pour lui, le dramaturge consiste essentiellement dans un compromis entre le « dit» et «l'écrit»: «Le texte est écrit non seulement pour être dit, mais encore, dans une certaine mesure, pour donner l'impression qu'il n'a jamais été écrit.»(Larthomas 1972, 278). Dans le texte dramatique, l'écrit précède le dit en fonction de la représentions, ce qui fait, selon Larthomas, le théâtre appartenir à la littérature, étant que la parole en action est déjà un texte, mais un texte joué devant nous.

Les chercheurs regroupés dans le courant de génétique textuelle analysent de plus en plus précisément l'avant texte «Les écrits qui précèdent et accompagnent la production du roman pour essayer de mieux cerner le travail de création littéraire » (Reuter 1997, 105). N’y a-t-il que le texte romanesque où le paratexte apparait ?

Pour nous, le texte théâtral, de sa part, pourrait aussi en témoigner en tant que texte dramatique. Les didascalies représentent un élément essentiel de la composition du texte dramatique. Elles incarnent ce qui n'est pas dit par les personnages. Tout texte théâtral comporte, comme c'est déjà dit, deux types de discours ; en premier, les dialogues que prennent en charge les personnages-acteurs, considérés comme l'essentiel du texte, en second, les didascalies que nous lisons autour de ces dialogues. Elles, en tant que discours narratif, prennent en charge la partie muette dans le théâtre, et sont adressées, en premier, au lecteur qui devrait commencer à planter le décor, l'espace, et les personnages sur sa scène mentale : «Il 
ne faut pas raconter des événements que lorsqu'il est impossible de représenter cet événement sur la scène, lorsque leur récit ajoute de l'intérêt à la pièce (...) » (Scherer 1966, 235). Les didascalies peuvent être d'ordre scénique ; présentation des personnages, noms, relations familiales, fonction et structure de la pièce, ce qui est commode au début de presque tout texte théâtral dont voici un exemple du médecin malgré lui :

SGANARELLE, mari de Martine. MARTINE, femme de Sganarelle. M. ROBERT, voisin de Sganarelle. VALÈRE, domestique de Géronte. LUCAS, mari de Jacqueline. GÉRONTE, père de Lucinde. JACQUELINE, nourrice chez Géronte, et femme de Lucas. LUCINDE, fille de Géronte. LÉANDRE, amant de Lucinde. THIBAUT, père de Perrin. PERRIN, fils de Thibaut, paysan. (Molière 2000, 16)

Il s'agit en gros de toute indication paratextuelle dirigeant le lecteur vers l'espace, le lieu, le temps, le tour de parole, le sentiment, l'émotion, et l'effet discursif dans le texte de théâtre. Genette a abordé la question du paratexte dans Seuils parus en 1987.

La paratextualité révèle les diverses relations qu'entretient le texte avec les écrits qui le composent. Il ne s'agit pas du contenu diégétique ou historique de l'œuvre, mais des écrits qui le précèdent et accompagnent sa composition, telle que notes, préface, titre, épigraphe, couverture, commentaire, autobiographe. Ces composantes sont effectivement indispensables pour le lecteur. Elles déterminent la compréhension et l'orientation initiale vers le texte et sa lecture: «le paratexte, sous toutes ses formes, est un discours fondamentalement hétéronome, auxiliaire, voué au service d'autre chose qui constitue sa raison d'être, et qui est le texte. » (Genette 1978, 9). Elles représentent également un outil technique pour la mise en scène des lieux, espaces, et temps où les événements se poursuivent. L'acte premier du roi qui s'amuse d'Hugo nous révèle un acte narratif d'un narrateur préparant la scène première au lecteur :

Une fête de nuit au Louvre. Salles magnifiques pleines d'hommes et de femmes en parure. Flambeaux, musique, danse, éclats de rire - des valets portent des plats d'or et des vaisselles d'émail; des groupes de seigneurs et de dames passent sur le thêâtre. - La fête tire à sa fin; l'aube blanchit les vitraux. Une certaine liberté règne ; la fête a un peu le caractère d'une orgie. - Dans l'architecture, dans les ameublements, dans les vêtements, le goût de la renaissance (Hugo 2008, 36)

L'insertion de certaines séquences narratives dans le corps dramatique a été codifiée après La Fronde par l'abbé d'Aubignac dans la pratique du théâtre, au chapitre III du livre IV intitulé « des narrations », où il répertorie trois types de narration dans le texte théâtral : les narrations d'exposition, les narrations de dénouement et les narrations incidentes (Aubignac 1957, 378-379). Ce qui nous importe le plus maintenant, ce sont celles d'exposition qui portent sur ce qui se passe avant que le rideau se lève, et se place au début afin de préparer à l'action et à sa réception.

Les éléments de didascalies servent ainsi de moyen narratif en provenance de l'extra diégétique pour remplir une valeur expressive. Elles apparaissent pour mettre en scène les sentiments, émotions, et caractères de visage des personnages, que nous ne ressentons que sur scène. Examinons ainsi certains 
aspects de didascalies rencontrés le plus souvent dans presque tout texte dramatique : «Il la regarde fixement. Elle se tait effrayée » (Hugo 2015, 5), « KUNZ, avec une sorte d'effroi. », «Les burgraves baissent la tête avec une sombre expression d'abattement, d'indignation et d'épouvante. Il poursuit» (Hugo 2009, 31-115).

Le lecteur doit ainsi vivre la chaleur et le rythme du dialogue et sentir l'effet du discours qu'il ne peut voir dans le texte, sauf sur scène où il voit et entend tout en direct. Mais, aucun sentiment ou émotion discursifs ne se révèle textuellement que par une intrusion narrative : une position que le narrateur choisit afin d'intégrer tous les aspects de la représentation théâtrale.

À partir du XIXe siècle, en particulier dans le drame romantique, les didascalies jouent un rôle important dans l'atmosphère théâtrale. Elles apparaissent, au début des scènes, sous forme des phrases nominales longues à fonction descriptive, présentative et de commentaire, ce qui ne parait que rarement dans le texte théâtral classique où nous ne rencontrons que ce qui a un rapport avec les actions et l'espace, mais bien raccourci. Le drame romantique d'Hugo nous laisse un bon exemple au début de la scène première de l'acte II de son Hernani :

Un patio du palais de Silva. À gauche, les grands murs du palais, avec une fenêtre à balcon. Au-dessous de la fenêtre, une petite porte. À droite et au fond, des maisons et des rues. - Il est nuit. - On voit briller çà et là, aux façades des édifices, quelques fenêtres encore éclairées. Un patio du palais de Silva. À gauche, les grands murs du palais, avec une fenêtre à balcon. Au-dessous de la fenêtre, une petite porte. À droite et au fond, des maisons et des rues. — Il est nuit. — On voit briller çà et là, aux façades des édifices, quelques fenêtres encore éclairées. (Hugo 2015, 25)

Cette longue didascalie qui débute la scène a son pareil, mais cette fois à la fin de la scène. Hugo parait dans son drame romantique encadrer les répliques de ses personnages par de narrations ayant pour objectif de mettre en scène le décor, le lieu, et le temps. Mais cette fois, nous tombons sur des narrations des objets qui composent un des éléments de l'intrigue dans une description minutieuse en focalisation externe, comme l'œil de la camera qui enregistre ce qui apparait devant les yeux du spectateur :

La porte du fond s'ouvre à deux battants. Entre doña Sol, en parure de mariée. Derrière elle, pages, valets, et deux femmes portant sur un coussin de velours un coffret d'argent ciselé, qu'elles vont déposer sur une table, et qui renferme un riche écrin, couronne de duchesse, bracelets, colliers, perles et brillants, pêle-mêle. Hernani, haletant et effaré, considère doña Sol avec des yeux ardents, sans écouter le duc (Hugo 2015, 47) (Je souligne).

À la lecture attentive de cette narration, il est à remarquer que son narrateur nous dit ce que son personnage va faire «qu'elles vont déposer sur une table». Le coffret contenant des bijoux va retrouver sa place sur la table. C'est ainsi un acte qui se passera dans quelque temps et que le narrateur choisit de dire au lecteur pour l'avertir d'avance et l'orienter dans sa réception de la scène. Selon les techniques narratives de Genette, il s'agit d'une prolepse qui tombe dans la même ligne de l'histoire et que l'on 
rencontre le plus souvent au récit ; voir le roman, le conte et la nouvelle dont voici un exemple de Gil Blas de Santillane de Lesage :

(...), Le ciel a daigné m'accorder deux enfants, dont l'éducation va devenir

l'amusement de mes vieux jours, et dont je crois pieusement être le père

(Lesage 745-746) (Je souligne).

Les didascalies se rangent au niveau de focalisation externe où le narrateur se prive d'entrer au fond de ses personnages et reste en observation de leurs actions externes et leur apparence. Il s'engage également à décrire de l'extérieur tout ce qui compose la scène. Mais que se passe-t-il lorsqu'il s'agit d'une infraction focale?

En dépit de toutes ces informations didascaliques en focalisation externe, le narrateur pourrait sortir de ce code et lance une information complémentaire dans un geste personnel. La richesse d'information ; un des procédés narratifs qu'adopte le narrateur pour qu'un lecteur puisse mieux imaginer le récit, et ainsi, pour nous maintenant, le texte dramatique.

Le narrateur des didascalies prend dans certaines situations une position et un avis personnel quant à son intrigue. Il semble quitter la focalisation externe, une vision objective et externe des personnages et des objets, et va jusqu'à atteindre la focalisation zéro dans un commentaire narratif extradiégétique. Ceci parait avoir l'objectif de prendre une position et de s'engager au côté de l'un de ses personnages. Molière fait, dans Tartuffe, un meilleur exemple d'un narrateur stigmatisant son personnage et prend position contre ses actes :

TARTUFFE : Je puis vous dissiper ces craintes ridicules,

Madame, et je sais l'art de lever les scrupules.

Le Ciel défend, de vrai, certains contentements ;

(C'est un scélérat qui parle.) (Molière 2000, 859)

Tartuffe, en tant que personnage jouant le rôle d'un faux dévot, est sévèrement stigmatisé, en fin de la réplique, par le narrateur de cette didascalie; un scélérat. C'est un témoignage écrit de niveau extradiégétique afin de dénoncer les friponneries couvertes de cette fausse dévotion au XVII Siècle. Dans le théâtre, un spectateur ne voit jamais cette formule et doit comprendre la scélératesse de Tartuffe. Un lecteur doit lui aussi découvrir ce faux dévot à travers sa lecture, mais cette fois, un narrateur décide avec exprès d'intervenir pour renforcer et affirmer ce que le lecteur du texte dramatique doit comprendre sur ce personnage ; un avertissement au lecteur.

Parfois, le narrateur des didascalies dans Tartuffe parait, au contraire, s'engager explicitement aux côtés de l'un de ses personnages. Il interrompt la réplique et insère un rappel au lecteur du type de personnage qui prend la parole. Un rappel narratif à objectif social et humain :

DORINE (...). Depuis que de Tartuffe on le voit entêté.

Il l'appelle son frère, et l'aime dans son âme

Cent fois plus qu'il ne fait mère, fils, fille, et femme. (...) 


\section{(C'est une servante qui parle.)}

Enfin il en est fou ; c'est son Tout, son héros ;

Il l'admire à tous coups, le cite à tout propos (...) (Molière 2000, 10)

Dorine, en tant que personnage - servant parait prendre dans le théâtre de Molière un rôle imprévu au temps classique; les valets et les servants ne doivent jamais se mettre en face de leurs maitres et les contrarier. Cette réplique, dans laquelle elle critique la conduite et l'attitude de son maitre (Orgon) dans un discours droit et direct, pousse le narrateur à intervenir au milieu et à nous dire que c'est une servante qui parle droitement sans crainte, alors que normalement, à cette époque - la, elle n'a aucun droit de critiquer et d'opposer l'avis de son maitre.

Parfois, le dramaturge lance dans son texte un surplus d'information qui ne tombe en aucune des faveurs de l'un de ses personnages. Il s'agit, au milieu des discours dramatiques, d'une petite intervention narrative en provenance de l'extradiégèse. Le drame romantique d'Hugo nous laisse un exemple évident à la scène VI de l'acte III d'Hernani :

Hernani, don Ruy Gomez, doña Sol. DON RUY GOMEZ, immobile et croisant les bras.

Voilà donc le paiement de l'hospitalité ! Voilà ce que céans notre hôte nous apporte !

Tous deux se détournent comme réveillés en sur saut. (Hugo 2015, 52)

Les deux séquences de didascalie qui encadrent le discours du personnage sont dominées par la focalisation externe d'un narrateur qui nous rapporte leurs états corporels et leurs actions ; (immobile et croissant les bras) (tous deux se détournent), mais l'acte de se détourner est explicitement accompagné d'une information ou bien d'un commentaire en focalisation zéro ayant pour objectif de refléter au lecteur la nature de détournement; ils les ressemble à un réveillé en sursaut pour que le lecteur imagine bien l'action. Les indications didascaliques nous indiquent normalement tout muet qui doit être représenté littéralement au lecteur. L'ajout d'un commentaire narratif n'a d'autre justification qu'un désir ardent du narrateur de se montrer médiateur et guide à son lecteur afin de bien visualiser la scène : «Certains éléments de paratexte s'adressent effectivement (ce qui ne signifie pas qu'ils l'atteignent) au public en général, c'est-à-dire à tout un chacun » (Genette 1978, 10)

Dans le texte narratif, l'on rencontre carrément différentes fonctions du narrateur à partir de son instance narrative. La fonction narrative est la base de toute médiation de narrateur en tant qu'une fiction à raconter. Mais, quand il renonce à cette fonction et assume une autre de régie, il parait proposer une dimension autre que celle de raconter une histoire; il repart du niveau diégétique au niveau extradiégétique afin de bien contrôler l'organisation de son récit et de diriger son lecteur. Moby Dick de Heman Melville nous donne un exemple :

Ce chapitre est aussi important que n'importe quel autre de ce livre, sans faire à proprement parler partie du récit. (Melville 2007, 360) 
Dans le texte dramatique, le régisseur pourrait aussi bien remplir cette fonction lors de son intervention didascalique. Comme c'est déjà dit, le script nécessite un outil de guidage lors de sa composition textuelle des éléments non verbaux; actions, décor, espace, temps, etc. Le narrateur régisseur trouve une chance de révéler à son lecteur l'organisation des scènes en question, ou peut-être celles qui suivent. Voir ces interventions métanarratives d'un narrateur régissant son texte dramatique : «Pendant toute la première partie de la scène, Triboulet a l'air de chercher, d'examiner, de fureter», (Hugo 2008,138), ou plus tard : «Pendant toute cette scène et la suivante, le roi et Maguelonne, toujours seul dans la salle basse, continuent de se faire des agaceries et de se parler à voix basse en riant. » (Hugo 2008, 117). Ou encore cet exemple pris d'Hernani «Pendant tout le commencement de la scène qui suit, les fanfares et les lumières éloignées s'éteignent par degrés ». (Hugo 2015, 91)

Le narrateur se réfère ainsi à son texte narratif dans un discours métanarratif. Il s'agit d'une indication de régie selon George Blin; un contrôle, en provenance de l'extradiégétique, qu'exerce le narrateur pour ainsi régir et marquer l'organisation et l'articulation interne de son récit. (Blin, cité par Genette 1972, 262)

\section{Incipit et prologue ; une valeur narrative}

Les études modernes sur la narratologie ont effectivement donné une importance à l'incipit du roman; les premiers mots qui orientent le lecteur à la compréhension du texte, ou peut-être la retardent. Le terme incipit vient du verbe latin incepere qui signifie commencer. Il s'agissait avant tout les premiers mots des manuscrits au moyen-âge, et devenus après l'invention de l'imprimerie les premières phrases d'une publication. C'est une structure narrative d'accès que nous ne pouvons isoler du récit qui s'en constitue.

Nous devons donc parler du seuil qui nous lance dans le corps du texte ${ }^{1}$ et représente un calme de récit et d'événements, lors duquel l'on se prépare au détail de l'action. Nous y sommes ainsi au contexte, dès que nous repérons la fin de l'incipit, des questions se posent ; et quoi après ? Que se passera-t-il ?

Le drame d'Hugo fait dans la majorité de sa composition une structure narrative préparant le lecteur à accéder au contexte dramatique. Les scènes des Burgraves sont précédées à la première partie d'une entrée intitulée (L'AÏEUL) ayant une valeur explicative et informative, racontée par un narrateur anonyme et extérieur à l'histoire :

L'ancienne galerie des portraits seigneuriaux du burg de Heppenheff. Cette galerie, qui était circulaire, se développait autour du grand donjon, et communiquait avec le reste du château par quatre grandes portes situées aux quatre points cardinaux. Au lever du rideau, on aperçoit une partie de cette galerie, qui fait retour et qu'on voit se perdre derrière le mur arrondi du donjon. À gauche, une des quatre grandes portes de communication. À droite, une haute et large porte communiquant avec l'intérieur du donjon, exhaussée sur un degré de trois marches et accostée d'une porte bâtarde (Hugo 2009, 19). 
Une voix anonyme décrit le lieu où apparaissent l'action, le château, les donjons, l'ancienne galerie et sa communication au château. Elle dirige la conception du lecteur sur le décor au lever de rideau. Au deuxième paragraphe, cette voix trouve une nécessité de préciser le temps de l'action pour ainsi justifier l'éclairage d'une partie du théâtre, et annoncer au lecteur le soir :

Au moment où le rideau se lève, le soir vient. La partie du château qu'on aperçoit par les archivoltes du promenoir au fond du théâtre semble éclairée et illuminée à l'intérieur, quoiqu'il fasse encore grand jour (Hugo 2009, 19-20).

Puis, le narrateur décide de nous mettre sur le seuil de l'action en concentrant son exposition sur les personnages qui devront se trouver à la scène première. Le bruit qui s'entend quelque part sur la scène est aussi justifié :

On entend venir de ce côté du burg un bruit de trompettes et de clairons, et par moments des chansons chantées à pleine voix au cliquetis des verres (...). Une femme, seule, vieille, à demi cachée par un long voile noir, vêtue d'un sac de toile grise en lambeaux, enchaînée d'une chaîne qui se rattache par un double anneau à sa ceinture et à son pied nu, un collier de fer autour du cou, s'appuie contre la grande porte, et semble écouter les fanfares et les chants de la salle voisine. (Hugo 2009, 20).

Cet incipit qui n'existait plus au théâtre classique n'a couvert qu'une page et demie et a atteint son objectif de mettre le lecteur sur la marche d'une pièce historique. Les trois éléments d'une compréhension élémentaire y sont bien évidents ; le lieu, le temps et enfin l'action. Varga voit ainsi cet aspect hétérogène du texte théâtral : "Il contient en général des éléments non dramatiques qui proviennent de l'exposition, c'est-à-dire, des renseignements concernant des événements antérieurs à l'action et que l'auteur fait raconter a quelqu'un en général au début de la pièce » (Varga 1988, 84)

Le dramaturge fait ainsi raconter tout ce qui n'est pas dramatique par une voix. Mais à qui appartient-elle ?

Il n'y a ni histoire ni récit sans narrateur qui, à la différence de sa façon de raconter en (Je) ou en (Il), reste un personnage fictif inventé par l'écrivain. Comme l'usage de l'incipit raconté par un narrateur anonyme, le dramaturge pourrait adopter une autre voix, mais cette fois d'un personnage qui n'a d'autre rôle que d'exposer et de présenter ; c'est le prologue.

Avant d'entreprendre le rôle et la fonction du prologue dans certaines pièces de théâtre tragiques où il apparait en particulier, il est indispensable de traiter l'étymologie de ce mot. Au latin (prologue) désigne le début d'une pièce de théâtre et au grec ancien il signifie (préambule). Le mot (prologue) est composé de deux mots à l'origine : (pro=avant), et (logue= texte), c'est donc l'avant-texte d'une œuvre, ou bien en France d'un Opéra. Il sert effectivement de prélude à une pièce dramatique. Nous choisissons Antigone d'Anouilh, une pièce où le rôle du prologue est innové par rapport à celui de Sophocle².

Le prologue fait le plus souvent le discours d'ouverture de la tragédie. Le spectateur ainsi que le lecteur sont bien préparés à l'action et n'en sont plus surpris, ils savent déjà. Ce prologue est l'énonciateur 
de lancement de la pièce et de son dénouement. Le malheur, la mort, le défi et le désespoir sont déjà informés dès le début par ce personnage qui n'a d'autre rôle que d'avertir afin d'éviter toute chance de surprise. Il sait donc déjà tout; le présent le passé, l'avenir et même l'intérieur des personnages qui peuplent la scène. Il pourrait se ranger aux côtés des narrateurs omniscients selon la terminologie de Genette. Il occupe une position appelée (intradiégétique) tant qu'il figure parmi les personnages de la pièce, mais qui n'a aucune communication avec eux. Il ressemble, en raison de la connaissance qu'il a sur les personnages et les événements avenir, à un dieu scénique qui apparait pour commenter, analyser, annoncer et prévoir une situation théâtrale.

Au début d'Antigone, nous entendons une voix narrative en provenance de l'exradiégèse, dont nous avons déjà parlé à propos de la didascalie mettant en scène le décor, le lieu, le temps et en particulier le personnage du prologue :

Un décor neutre. Trois portes semblables. Au lever du rideau, tous les personnages sont en scène. Ils bavardent, tricotent, jouent aux cartes.

Le Prologue se détache et s'avance (Anouilh 1987, 9)

C'est le narrateur premier qui va ensuite déléguer l'acte de raconter au prologue qui, de sa part, se détache effectivement, s'avance et s'adresse au public comme narrateur second au niveau métadiégétique. Il fait son intervention et assure sa fonction d'exposition et de présentation des personnages :

Voilà. Ces personnages vont vous jouer l'histoire d'Antigone. Antigone, c'est la petite maigre qui est assise là-bas, et qui ne dit rien (...)

Le jeune homme avec qui parle la blonde, la belle, l'heureuse Ismène, c'est Hémon, le fils de Créon. Il est le fiancé d'Antigone. (...)

Cet homme robuste, aux cheveux blancs, qui médite là, près de son page, c'est Créon. C'est le roi. (...)

La vieille dame qui tricote, à côté de la nourrice qui a élevé les deux petites, c'est Eurydice, la femme de Créon (...)

Ce garçon pâle, là-bas, au fond, qui rêve adossé au mur, solitaire, c'est le Messager (...)

Enfin les trois hommes rougeauds qui jouent aux cartes, leurs chapeaux sur la nuque, ce sont les gardes (...) (Anouilh 1987, 9-10).

Richardson voit que, même au théâtre, l'on pourrait avoir un narrateur au niveau second qui prend en charge l'acte de raconter et change ainsi le rythme narratif : «(...) Le théâtre est d'autant plus capable de marquer ces différences de ton et de temporalité, usant d'un narrateur entrant et sortant successivement de son personnage pour s'adresser tantôt au public tantôt aux autres acteurs. » (Richardson cité et traduit par Hennaut). La préposition (voilà) prononcé par le prologue désigne au public le décor, les personnages et tout ce qui est spatio-temporel. Cette présentation est nécessaire au spectateur car il n'a pas de chance de lire le paratexte initial faisant une démonstration des personnages (nom et fonction). Dans le texte, ce 
rôle de prologue est aussi nécessaire pour le commentaire, l'analyse et les informations sur les scènes présentées et celles à venir.

Une des fonctions qu' assure le prologue est celle de communication au public qui, de sa part, voit en lui le guide théâtral :

(...) Ces personnages vont vous jouer l'histoire d'Antigone (...), mais ils vous empoigneront les accusés le plus tranquillement du monde tout à l'heure (...). Et maintenant que vous les connaissez tous, ils vont pouvoir vous jouer leur histoire. (Anouilh 1987, 9-10).

L'énonciation dramatique est toujours double. Il y a en fait deux destinataires au discours dans le texte théâtral, comme le confirme Ubersfeld dans son Lire le théâtre, il y a "un discours rapporteur » émis par le personnage par la médiation de l'acteur, et qui a pour destinataire un autre personnage, et un «discours rapporteur» émis par l'auteur et qui a pour destinataire le public (Ubersfeld 1978). Ce qui fait du prologue une porte - parole de l'auteur afin d'assurer un message d'informer le destinataire. Cette fonction de communication est bien évidente dans l'art de roman qui use énormément de cette technique :

Comment ? Pardonnez-moi, je pensais à autre chose. Je vous reverrai demain, sans doute. Demain, oui, c'est cela. Non, non, je ne puis rester (...) (Camus 2011, 23).

La structure du prologue remplit aussi une fonction de présentation de l'histoire que celle d'exposition des personnages. Une présentation actionnelle qui fait de lui un organisateur narratif de l'histoire.

Cette organisation de l'ordre des événements, analysée selon Genette sous le terme de l'anachronie narrative, et appliquée le plus souvent sur le texte romanesque, est en deux types; l'anachronie par anticipation appelée «prolepse » qui consiste à raconter ou à évoquer à l'avance un événement ultérieur, et l'anachronie par rétrospection appelée « analepse » ou «flash-back » au cinéma, qui consiste à raconter ou à évoquer après coup un événement antérieur (Genette 1972, 105-115)

Être un personnage - narrateur ayant une faculté omnisciente range ce prologue aux côtés des narrateurs omniscients de Balzac, qui, de leur part, contrôlent l'histoire qu'ils racontent et paraissent savoir profondément tout ce qui entoure les événements et les personnages qui peuplent l'histoire.

L'histoire d'Antigone est une suite à un événement capital qui fait l'enjeu d'une tragédie et la cause d'une fatalité dramatique. Le lecteur ainsi que le spectateur ont effectivement besoin d'une voix qui leur raconte l'événement antérieur de l'histoire racontée. Le prologue prend, de sa part, cette tache dans une « analepse » ou bien un flash-back qui justifie la suite de l'histoire :

Elle commence au moment où les deux fils d'CEdipe, Étéocle et Polynice, qui devaient régner sur Thèbes un an chacun à tour de rôle, se sont battus et entre-tués sous les murs de la ville, Étéocle lainé, au terme de la première année de pouvoir, ayant refusé de céder la place à son frère. Sept grands princes étrangers que Polynice avait gagnés à sa cause ont été défaits devant les sept portes de Thèbes. Maintenant la ville est sauvée, les deux frères ennemis sont morts et Créon, le roi, a ordonné 
qu'à Étéocle, le bon frère, il serait fait d'imposantes funérailles, mais que Polynice, le vaurien, le révolté, le voyou serait laissé sans pleurs et sans sépulture, la proie des corbeaux et des chacals. Quiconque osera lui rendre les devoirs funèbres sera impitoyablement puni de mort. (Anouilh 1987, 12-13)

Cette analepse de type externe, dont la portée et l'amplitude se sont survenues avant le début de l'histoire d'Antigone, fait du prologue un rôle narratif qui devrait nous raconter l'antécédent de cet événement sans lequel nous ignorerons l'origine de ce défi d'Antigone à son oncle Créon et à sa loi.

Comme tout narrateur omniscient, ici le cas exceptionnel, il anticipe les événements et nous rend au courant de ce qui va se passer dans une prolepse interne dont la portée et l'amplitude sont incluses dans l'histoire :

Elle pense quelle va être Antigone tout à l'heure, qu'elle va surgir soudain de la maigre jeune fille noiraude et renfermée que personne ne prenait au sérieux dans la famille et se dresser seule en face du monde, seule en face de Créon, son oncle, qui est le roi. Elle pense qu'elle va mourir (Anouilh 1987, 9)

Il fait aussi allusions aux actions prédestinées sur la vie d'Hémon et sur son existence à venir :

Il ne savait pas qu'il ne devait jamais exister de mari d'Antigone sur cette terre et que ce titre princier lui donnait seulement le droit de mourir. (Anouilh 1987, 11)

Ces informations initiales dépassent la conscience des personnages eux-mêmes. L'anticipation du prologue va jusqu'à annoncer le dénouement en désignant le personnage qui va annoncer la mort d'Hémon qu'il a prévue tout à l'heure, c'est le messager :

Ce garçon pâle, là-bas, au fond, qui rêve adossé au mur, solitaire, c'est le Messager.

C'est lui qui viendra annoncer la mort d'Hémon tout à l'heure (Anouilh 1987, 12)

Ce discours du prologue se range ainsi aux côtés des incipits ayant pour fonction d'informer le lecteur ou le spectateur et de nouer le contrat de lecture et de compréhension initiale de la pièce. Sa présence pourrait être équivalente de celle du narrateur qui débute son récit par un incipit informatif.

\section{Conclusion}

La distinction des anciens entre les modes, diégétique et mimétique, ne parait pas effective dans le monde thêâtral, surtout dans le théâtre moderne où des instances narratoriales encadrent les actions et orientent le lecteur vers la réception de l'action; ses antécédents et, peut-être, ses événements à venir.

Le script (texte dramatique), n'est ainsi qu'une traduction narrative d'une performance (représentation théâtrale). Le discours des personnages constitue une instance différente de celle du discours narratif de narrateur. Ce dernier crée un cadre narratif afin d'accomplir le monde dramatique dans un registre énonciatif doté d'une instance narratoriale autonome. Ainsi le narrateur des didascalies dans le texte théâtral assure-t-il une activité de metteur en scène d'action, d'événements, de sentiments et parfois de prise de position. 
L'acte de mettre en intrigue exige une unité entre le dramatique et le narratif. L'histoire, représentée par le biais des personnages, révèle des relations dramatico-narratives sans lesquelles les actions seraient désertes.

Enfin, le théâtre, comme le roman, la nouvelle et même le film, établit effectivement, à des degrés différents, des affinités avec la narration et la description en raison de l'actualisation de l'intrigue par des éléments narratifs médiateurs. Le discours des personnages incarnés dans le texte dramatique revendique une certaine médiation qui les place dans un ordre d'événements et d'action enchaînés. Les narrations dans le théâtre sont ainsi d'importance capitale pour que le développement logique des actions, ainsi que la réception de l'intrigue dramatique, se poursuive. 


$$
\begin{aligned}
& \text { السرد في النص المسرحي: وساطة سردية } \\
& \text { بشار سامي يشوع } \\
& \text { قسم اللفة الفرنسية، الجامعة المستنصرية، العراق }
\end{aligned}
$$

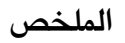

تنطلق المفاهيم الأولى للأولين التي تناولت تحليل السرد والنص المسرحي، من التمييز بين مفهوم (Mimesis) وهو فن العرض، و (Diégesis)، وهو فن القص. يُنظر الى المسرح من الصفة الاستعراضية لبنائه. إذ يتم تصوير القصة دون أن تُروى. حيث يبدو ان الدراسات الأدبية والنقدية للقدماء، أمثال أرسطو وأفلاطون، قد حددت المسرح ضمن نطاق العرض

لا يبدو هذا أن التمايز بين الإثنين تام في العالم المسرحي. فالنص الدرامي ليس إلا ترجمة سردية للتمثل المسرحي ومن ذلك قد تنثأ العلاقة بين النص المسرحي والسرد. تكمن إثكالية دراستنا في أن بمقدور النص الدرامي أو المسرحي أن يتضمن جوانب سردية انطلاقًا من المفهوم الأساسي لوظائف الراوي والوضع السردي. إذ تصبح عملية البحث عن راوٍ في

$$
\text { النص المسرحي ضرورة. }
$$

ونجد أوضاعًا سردية، غير تلك الدراميّة التي تقوم بها الثخصيات، تعمل على تأطير الأحداث، وتقود القارئ إلى فهٍِ وتلقِ كاملٍ للعمل الادبي. ويحتل خطاب الشخصيات موضعًا في مستوى سردي غير ذلك الذي يحتله الراوي الذي يتخذ في أغلب الأحيان، موقعًا خارج حكائي، ويخلق إطارًا سرديًا يهدف إلى كمال صورة العالم الدرامي. إن ملفوظات الإخراج المسرحي وكتابات ما وراء النص هي مستويات لفظية تشكل وضعًا سرديًا مستقلاً. فالأحداث والمشاعر وتعبيرات الوجه صامتة، إلا أنها تجد في هذا الوضع السردي للراوي وسيلة لإخراج المحيط والبيئة الدرامية. لذلك، بمقدور النص المسرحي احتواء مظاهر سردية انطلاقًا من راوٍ يؤدي وظائف الوساطة السردية، ويؤسس فعليًا مقاربة مع السرد والوصف بغية إيصال الإطار والبيئة والمحيط غير اللفظي ونقله. الكلمات المفتاحية: الراوي، المسرح، الحكي، الاستهلال، ملفوظات الإخراج المسرحي، كتابات ما وراء النص. 


\section{Notes de fin}

${ }^{1}$ Il y a aussi des incipits secondaires, rapportés aux débuts des chapitres, qui renforcent l'incipit principal. Celui-ci pourrait durer pour tout un chapitre ou peut être des chapitres. Il déplace le destinataire de la réalité à la fiction.

${ }^{2}$ Anouilh s'inspire de Sophocle dans son Antigone, mais il accorde des caractéristiques nouvelles à son prologue; entrée en scène, présentation des personnages, registre de langue, anachronismes et d'autres aspects scéniques qui le rend innové par rapport à celui de Sophocle.

\section{Références}

Anouilh, Jean. 1987. Antigone. éd. La Table Ronde. Paris.

Aubignac, Abbé de. F. Helbin. 1657. La Pratique du Théâtre. Antoine de Somma ville. Paris.

Buffard-Moret, Brigitte. 2000. Introduction à la stylistique. Nathan, Lettres. Paris.

Camus, Albert. 2011. La Chute. éd. Ebooks libres et gratuits. Paris.

Genette, Gérard. 1969. Figures II. éd. Du Seuil, coll. Points. Paris.

Genette, Gérard. 1972. Figures III. éd. Seuil, coll. Poétique. Paris.

Genette, Gérard. 1978. Seuils. Seuils, poétiques. Paris.

Genette, Gérard. 1983. Nouveau Discours du récit. éd. Du Seuil. coll. poétique.

Hennaut, Benoît. «Narratologie et écritures théâtrales: quel dialogue possible? », Cahiers de Narratologie [En línea], 24 | 2013, Publicado el 17 septiembre 2013, consultado el 08 mayo 2020. URL: http://journals.openedition.org/narratologie/6669; DOI :https://doi.org/10.4000/ narratologie. 6669

Hugo, Victor. 2008. Le roi s'amuse. Ebooks libres et gratuits. Paris.

Hugo, Victor. 2009. Les Burgraves. éd, Ebooks libres et gratuits. Paris.

Hugo, Victor. 2015. Hernani. éd. Théâtre Classique. Gwénola, Ernest et Paul Fièvre.

Larthomas, Pierre. 1972. Le Langage Dramatique. A. Collin. Paris.

Lesage, Alain René. Gil Blas de Santillane. éd. Lirvrot gratis. coll. Romans/Nouvelles. (En ligne)

Melville, Herman. 2007. Moby Dick. éd. Ebook libres et gratuits, classique, coll. Numerique. Paris.

Molière. 2000. CEuvres Complètes I, Tartuffe. éd. EBooks France.

Molière. 2000. Euvres complètes II, Le médecin malgré lui. éd. EBooks France.

Reuter, Yves. 1997. L'Analyse du Récit. DUNOD. Paris.

Richardson, Brian. 1988. Point of View in Drama, Diegetic Monologue, Unreliable Narrators and the Authors Voice on Stage, in Comparative Drama, vol. 22, $\mathrm{n}^{\circ} 3$.

Scherer, Jacques. 1966. La Dramaturgie Classique en France. Paris: A. G. Nizet, s.d.

Ubersfeld, Anne. 1978. Lire le théâtre. éd. Sociales.

Varga, Kibédi Aron. Scènes et lieux de la tragédie. In: Langue française, nº79, 1988. Rhétorique et littérature.pp.82-95.www.persee.fr/doc/lfr_0023-8368_1988_num_79_1_4754,

DOI: https://doi.org/10.3406/lfr.1988.4754 